
UNDER CONTRACT DE-AC02-76CH03073

PPPL-3597

PPPL-3597

UC-70

\title{
Physics of Compact Advanced Stellarators
}

by

M.C. Zarnstorff, L.A. Berry, A. Brooks, E. Fredrickson, G.-Y. Fu, S. Hirshman, S. Hudson, L.-P. Ku, E. Lazarus, D. Mikkelsen, D. Monticello, G.H. Neilson,

N. Pomphrey, A. Reiman, D. Spong, D. Strickler, A. Boozer, W.A. Cooper,

R. Goldston, R. Hatcher, M. Isaev, C. Kessel, J. Lewandowski, J. Lyon,

P. Merkel, H. Mynick, B.E. Nelson, C. Nuehrenberg, M. Redi,

W. Reiersen, P. Rutherford, R. Sanchez,

J. Schmidt, and R.B. White

August 2001

N/

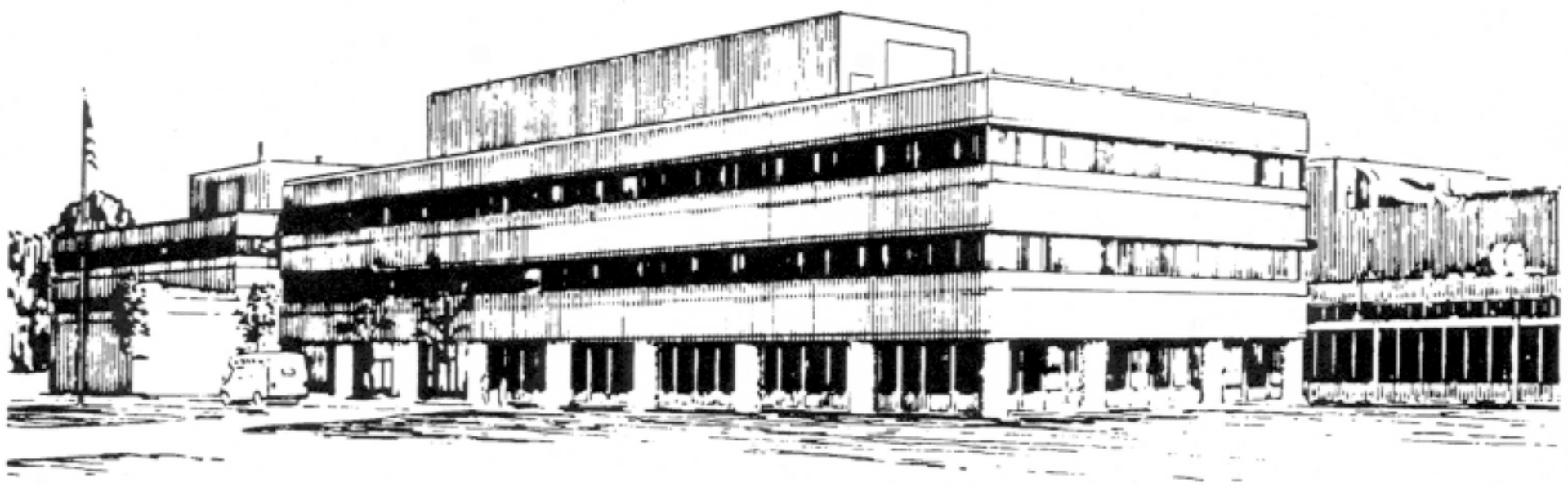

PRINCETON PLASMA PHYSICS LABORATORY PRINCETON UNIVERSITY, PRINCETON, NEW JERSEY 


\section{PPPL Reports Disclaimer}

This report was prepared as an account of work sponsored by an agency of the United States Government. Neither the United States Government nor any agency thereof, nor any of their employees, makes any warranty, express or implied, or assumes any legal liability or responsibility for the accuracy, completeness, or usefulness of any information, apparatus, product, or process disclosed, or represents that its use would not infringe privately owned rights. Reference herein to any specific commercial product, process, or service by trade name, trademark, manufacturer, or otherwise, does not necessarily constitute or imply its endorsement, recommendation, or favoring by the United States Government or any agency thereof. The views and opinions of authors expressed herein do not necessarily state or reflect those of the United States Government or any agency thereof.

\section{Availability}

This report is posted on the U.S. Department of Energy's Princeton Plasma Physics Laboratory Publications and Reports web site in Calendar Year 2001. The home page for PPPL Reports and Publications is: http://www.pppl.gov/pub_report/

DOE and DOE Contractors can obtain copies of this report from:

U.S. Department of Energy

Office of Scientific and Technical Information

DOE Technical Information Services (DTIS)

P.O. Box 62

Oak Ridge, TN 37831

Telephone: (865) 576-8401

Fax: (865) 576-5728

Email: reports@adonis.osti.gov

This report is available to the general public from:

National Technical Information Service

U.S. Department of Commerce

5285 Port Royal Road

Springfield, VA 22161

Telephone: 1-800-553-6847 or

(703) 605-6000

Fax: (703) 321-8547

Internet: http://www.ntis.gov/ordering.htm 


\section{Physics of Compact Advanced Stellarators.}

M.C. Zarnstorff ${ }^{1}$, L.A. Berry ${ }^{2}$, A. Brooks ${ }^{1}$, E. Fredrickson ${ }^{1}$, G.-Y. Fu ${ }^{1}$, S. Hirshman ${ }^{2}$, S. Hudson ${ }^{1}$, L.-P. Ku ${ }^{1}$, E. Lazarus ${ }^{2}$, D. Mikkelsen ${ }^{1}$, D. Monticello ${ }^{1}$, G.H. Neilson ${ }^{1}$, N. Pomphrey ${ }^{1}$, A. Reiman ${ }^{1}$, D. Spong ${ }^{2}$, D. Strickler ${ }^{2}$, A. Boozer ${ }^{3}$, W.A. Cooper ${ }^{4}$, R. Goldston ${ }^{1}$, R. Hatcher ${ }^{1}$, M. Isaev ${ }^{5}$, C. Kessel $^{1}$, J. Lewandowski $^{1}$, J. Lyon $^{2}$, P. Merkel $^{6}$, H. Mynick ${ }^{1}$, B.E. Nelson ${ }^{2}$, C. Nuehrenberg ${ }^{6}$, M. Redi $^{1}$, W. Reiersen ${ }^{1}$, P. Rutherford ${ }^{1}$, R. Sanchez ${ }^{7}$, J. Schmidt ${ }^{1}$, and R. B. White ${ }^{1}$.

\footnotetext{
${ }^{1}$ Princeton Plasma Physics Laboratory, Princeton, NJ 08543 USA

${ }^{2}$ Oak Ridge National Laboratory, Oak Ridge, TN 37831 USA

${ }^{3}$ Columbia University, New York, NY 10027 USA

${ }^{4}$ Ecole Polytechnique Federale de Lausanne, Lausanne, Switzerland

${ }^{5}$ Kurchatov Institute, Moscow, Russia

${ }^{6}$ Max Planck Institute for Plasma Physics,Greifswald, Germany

${ }^{7}$ Universidad Carlos III de Madrid, Spain
}

email contact of first author: zarnstorff@pppl.gov 
Abstract. Compact optimized stellarators offer novel solutions for confining high-beta plasmas and developing magnetic confinement fusion. The 3D plasma shape can be designed to enhance the MHD stability without feedback or nearby conducting structures and provide drift-orbit confinement similar to tokamaks. These configurations offer the possibility of combining the steady-state low-recirculating power, external control, and disruption resilience of previous stellarators with the low-aspect ratio, high beta-limit, and good confinement of advanced tokamaks. Quasi-axisymmetric equilibria have been developed for the proposed National Compact Stellarator Experiment (NCSX) with average aspect ratio 4 - 4.4 and average elongation $\sim 1.8$. Even with bootstrap-current consistent profiles, they are passively stable to the ballooning, kink, vertical, Mercier, and neoclassical-tearing modes for $\beta$ $>4 \%$, without the need for external feedback or conducting walls. The bootstrap current generates only $1 / 4$ of the magnetic rotational transform at $\beta=4 \%$ (the rest is from the coils), thus the equilibrium is much less non-linear and is more controllable than similar advanced tokamaks. The enhanced stability is a result of 'reversed' global shear, the spatial distribution of local shear, and the large fraction of externally generated transform. Transport simulations show adequate fast-ion confinement and thermal neoclassical transport similar to equivalent tokamaks. Modular coils have been designed which reproduce the physics properties, provide good flux surfaces, and allow flexible variation of the plasma shape to control the predicted MHD stability and transport properties. 


\section{Introduction}

Magnetic plasma confinement in toroidal geometries is well established and promising as a basis for future fusion reactors. Tokamaks, and other axisymmetric configurations, produce the confining poloidal magnetic field by toroidal currents in the plasma, which are typically generated inductively. Tokamaks have demonstrated excellent short-pulse plasma performance in "compact" geometries, with aspect ratios (ratio of the plasma major radius to the average minor radius) usually less than 4. Stellarators use three-dimensional magnetic fields generated by coils to produce some or all of the confining poloidal magnetic field. Stellarators have demonstrated levels of performance approaching those of tokamaks, but generally at aspect ratios in the range of 6-12.

Since stellarators can produce all components of the magnetic field directly from external coils, they are intrinsically well suited for steady state operation and do not require external current drive systems. In addition, stellarators typically do not experience disruptive terminations (disruptions) of the plasma. For example, both the W7-A [1] and Cleo [2] experiments were able to eliminate disruptions at the density limit and when passing through edge $\mathrm{q}=2$ (rotational transform $\mathfrak{t}=0.5$ ) by the addition of small amounts of externally (coil) gen-

erated rotational transform to plasmas with substantial parallel plasma currents. Recent hybrid experiments on W7-AS [3] with induced toroidal current have generated disruptions at edge $t \sim 0.5(q \sim 2)$ when the plasma current profile is analyzed to be unstable to global tearing modes (extending from center to edge). This instability is well understood in both stellarators and tokamaks, and can be avoided either by design or care in experiment operation. Extending the study of stellarator stability and disruption avoidance to high- $\beta$ and low aspect ratio awaits new experiments. 
As in tokamaks, the shape of the 3D MHD equilibrium determines the physics properties of a stellarator. Three-dimensional equilibria offer many more degrees of freedom than are available for axisymmetric configurations and this additional shaping flexibility can be used to tailor the equilibrium to obtain desired physics properties. This was first systematically exploited in the development of the "Advanced Stellarator" (AS) concept [4], in which a stellarator configuration was numerically optimized to realize good equilibrium, stability, and transport properties, using theoretical/numerical models. The AS optimization approach produced the designs for the Wendelstein 7-AS and Wendelstein 7-X [5] (under construction) experiments in Germany.

Historically, the major challenge for stellarators has been to provide acceptable drift-orbit confinement, allowing low neoclassical transport losses and adequate fast-ion confinement. This is due to the fully three-dimensional shape, which has no ignorable coordinates and thus no conserved canonical momenta for drift orbits. As a consequence, the radial excursion of drift orbits is not necessarily bounded as it is in axisymmetric systems. In addition, a general 3-D magnetic field will have a strong modulation in the magnitude of $\mathrm{B},|\mathrm{B}|$, in every direction, producing strong plasma flow damping in all directions. Two strategies were identified [6] to provide adequate drift-orbit and neoclassical confinement, and have become practical through numerical optimization. The first strategy minimizes the poloidal variation of the magnetic field strength. This minimizes the Pfirsch-Schlüter and bootstrap currents [7], and reduces the radial component of the $\nabla \mathrm{B}$ drift. This strategy was developed in the design of the Wendelstein $7-\mathrm{X}$, with aspect ratio 10.6 , where the orbit confinement was specifically optimized to minimize the bootstrap current. This strategy, partially optimized, also underlies the design of Wendelstein 7-AS. It is being further developed at low aspect ratio in the design of the QPS experiment [8] 
The second strategy for 3D drift-orbit confinement is called 'quasi-symmetry', which is based upon work by Boozer [9] showing that drift-orbit topology and neoclassical transport depends only on the variation of $|\mathrm{B}|$ within a flux surface, not on the dependence of the vector components of B. This was used by J. Nuehrenberg [10,11] and P. Garabedian [12] to develop stellarators that, while three-dimensional in Euclidean space, have a direction (either helical or toroidal) of approximate symmetry of $|\mathrm{B}|$ in (Boozer) flux coordinates. Quasisymmetric configurations have drift-orbits similar to equivalent symmetric configurations, and thus similar neoclassical transport. Rotation in the quasi-symmetric direction is also undamped, as in a symmetric configuration. The first experimental test of quasi-symmetry is the Helically Symmetric eXperiment (HSX) now operating at the University of Wisconsin [13].

In parallel, there have been tremendous advances in the understanding of tokamak experiments and the ability to manipulate tokamak plasmas. There has been a general confirmation of ideal MHD equilibrium and stability theory and neoclassical transport theory. Methods for stabilizing and manipulating turbulent transport (particularly for ions) have been developed, allowing the elimination of anomalous ion-thermal and particle transport, and reduction of anomalous electron thermal transport. There is a general understanding of the importance of flow-shear stabilization as a mechanism for stabilizing ion turbulence. In addition, there are a theoretical predictions that undamped turbulence-generated flows (zonal flows) [14] are significant in saturating turbulent transport at the levels observed.

Quasi-axisymmetric stellarators offer novel solutions for confining high- $\beta$ plasmas by combining the best features of tokamaks and stellarators. They offer the possibility of combining the steady-state low-recirculating power, external control, and disruption resilience of the stellarator with the low aspect ratio, high beta-limit and good confinement of the ad- 
vanced tokamak. Using the 3D shaping flexibility available in a stellarator, configurations can be designed that are MHD stable without nearby conducting structure, require no current drive at high- $\beta$, and have good orbit confinement. The quasi-axisymmetry gives good orbit and neoclassical confinement, similar to equivalent tokamaks, and reduced damping of toroidal rotation. The reduced damping may allow manipulation of the radial electric field via driven rotation and the full development of zonal flows, similar to tokamaks. In addition, quasi-axisymmetric plasmas have significant bootstrap current, reducing the rotational transform required from the external coils. The rotational transform profile produced by the 3D shaping and bootstrap current can be designed to monotonically increase towards the plasma edge, like the core region of a 'reversed shear' advanced tokamak. This is predicted to stabilize neoclassical tearing modes, reduce equilibrium islands, and stabilize trappedparticle driven modes. Quasi-axisymmetry is well suited for the design of low aspect ratio configurations, since low aspect ratio forces the $n=0, m>1$ Fourier coefficients of the magnetic field strength to be dominant, where $n$ and $m$ are the toroidal and poloidal mode numbers, respectively. Low aspect ratio configurations are attractive in order to minimize the cost of near term experiments and the capital cost of possible future power plants.

These configurations are being studied for the design of the National Compact Stellarator Experiment (NCSX). It is proposed to study the physics of the beta limit in compact stellarators, the role of 3D shaping and externally generated transform in disruptions, and the ability to operate reliably without disruptions at the beta-limit with low collisionality and bootstrap current consistent profiles. It will also test quasi-axisymmetric reduction of neoclassical transport, the residual flow damping, effects on turbulence, and the ability to induce enhanced confinement. Compact quasi-axisymmetric stellarators are also being investigated for the design of the CHS-qa experiment [15]. 


\section{Configuration Design \& Stability}

The basic procedure used is similar to that developed for Wendelstein 7-X. A target fixedboundary plasma equilibrium is designed to have the desired physics properties. Coils are then designed to reproduce the target equilibrium and to generate good flux surfaces. The flexibility of the coils is tested by examining free-boundary equilibria with different profiles or different desired physics properties.

The target plasma configuration is designed by numerical optimization of the 3D plasma boundary shape to achieve the desired physics properties, such as transport and MHD stability. For these studies, the plasma has been optimized to be quasi-axisymmetric, ideal MHD stable at $\beta=4.25 \%$, have a monotonically increasing $\mathrm{t}$ profile (decreasing q profile), and be consistent with the bootstrap current. The optimizer calculates candidate 3D equilibria using VMEC [16] and then directly evaluates kink stability (for $n \leq 7$ using TERPSICHORE [17]), ballooning stability (using COBRA[18] and TERPSICHORE), the bootstrap current, and plasma transport. Several methods have been implemented for evaluating the transport characteristics, including the magnitude of the non-symmetric Fourier components of the magnetic field strength, the effective magnetic ripple [19], Monte-Carlo evaluation of fastion confinement, and a DKES [20] calculation of mono-energetic particle transport. In addition, the optimizer can evaluate approximate coil characteristics (using NESCOIL [21]) to encourage realizable configurations. 
A wide region of 3D configuration space was explored, including aspect ratios from 3 to 5 , edge rotational transform from 0.47 to 0.78 , rotational transform fraction due to $3 \mathrm{D}$ shaping from $50 \%$ to $80 \%$, and 2 to 4 periods. A broad pressure profile was assumed, within the range observed in existing stellarator experiments. The current profile was taken as the calculated bootstrap current, increased by $10 \%$ to provide margin against profile variation effects. A flat density profile was assumed for evaluating the bootstrap current. All of the configurations explored have large axisymmetric (or toroidal average) elongation and triangularity, to enable good stability to ballooning and kink modes. The vertical mode $(n=0)$ is calculated to be robustly stable [22], allowing consideration of average elongations up to 3 . A large number of interesting configurations were found with good properties. These were then evaluated for flux surface quality, coil current density and complexity, and fast ion confinement. To date, satisfactory coil designs have not been found for very large average elongations or very low aspect ratios.

Figure 1 shows the plasma boundary shape for the configuration adopted for the NCSX design. It has 3 field periods and an average aspect ratio $\langle A\rangle=R /\langle a\rangle=4.4$, where $R$ is the major radius and $\langle\mathrm{a}\rangle$ is the average minor radius. This configuration is calculated to be passively stable to the ballooning, low-n external and internal kink, and vertical instabilities up to $\beta=4.1 \%$, without need for a conducting wall or active feedback systems. The rotational transform profile increases from 0.4 on axis to 0.66 near the plasma edge, dropping to 0.65 at the plasma edge. Approximately $1 / 4$ of the edge rotational transform is from the bootstrap current and 3/4 is from the external coils. No external current drive is required. The bootstrap current is substantially smaller than in an equivalent advanced tokamak, due to the large transform from the coils. Due to the dominance of the coil generated rotational trans- 
form, the equilibrium is less sensitive to the pressure profile shape than in an advanced tokamak. This should allow control of the equilibrium via the external coils.

The kink stability is calculated to be due to the high rotational transform, reduced bootstrap current density, and the spatial variation of the local magnetic shear [23]. A systematic convergence study of the stability of equilibrium shown in Fig. 1 using TERPSICHORE revealed a weak $n / m=11 / 17$ instability localized to the plasma edge. Stability for all modes through $n \leq 20$ was achieved by a small modification of the plasma boundary shape. The passive vertical stability appears to be due to the substantial rotational transform produced by the external coils. Due to the rising rotational transform profile, neoclassical tearing modes are theoretically stable over all but the plasma edge. Relative to earlier designs $[24,25]$ this configuration has higher rotational transform, higher average elongation and triangularity, simpler coils, and better quasi-axisymmetry (less helical ripple). The improved quasiaxisymmetry produced a marked improvement in the calculated neoclassical energy confinement and fast-ion orbit confinement.

The toroidally averaged shape is similar to an advanced tokamak, with average elongation of 1.8 and an inside indentation of $9 \%$. For this average shape, an equivalent current $I_{P}^{\text {Equiv }}$ can be defined as the current required to match the stellarator edge rotational transform in a tokamak with the same average shape. NCSX is envisioned to have $\mathrm{R}=1.42 \mathrm{~m},\langle\mathrm{a}\rangle=0.33 \mathrm{~m}$, and $\mathrm{B}$ up to $1.7 \mathrm{~T}$ (at full external rotational transform). For these parameters, $I_{P}^{\text {Equiv }}=0.71$ MA. Evaluating $\beta_{N}^{\text {Equiv }}=\beta /\left(I_{P}^{\text {Equiv }} / a B\right)$ gives $\beta_{N}^{\text {Equiv }}=2.5$ for $\beta=4.1 \%$. Compared to advanced tokamaks, only moderate $\beta_{N}^{\text {Equiv }}$ is required due to the large $I_{P}^{\text {Equiv }}$ from the coilgenerated rotational transform. 
The quality of the flux-surfaces for this configuration has been evaluated using the PIES equilibrium code [26]. Figure 2(a) shows the calculated fixed-boundary equilibrium flux surfaces for the equilibrium of Fig. 1, showing a significant $n / m=3 / 5$ island, with a width of $\sim 10 \%$ of the minor radius and a smaller $\mathrm{n} / \mathrm{m}=3 / 6$ island in the core. These calculations do not include neoclassical-healing effects, from suppression of the bootstrap current in the island.

An analytic estimate of the neoclassical-healing gives an expected island width of $<5 \%$ of the plasma minor radius.

These islands have been removed from the equilibrium by modification of the plasma boundary shape [27]. A series of (short) PIES calculations are used to measure the change in island widths due to perturbations of resonant Fourier components of the plasma boundary shape. The calculation of the resonant fields and island widths is via construction of quadratic-flux minimizing surfaces [28]. The coupling matrix is inverted to give the change in boundary shape required to remove the observed equilibrium islands. Using this algorithm, the $\mathrm{n} / \mathrm{m}=3 / 4,3 / 5,3 / 6$, and $3 / 7$ Fourier components of the boundary minor radius were perturbed by $4.2,1.4,3.2$, and $-1.1 \mathrm{~mm}$, respectively. The resulting equilibrium, see Fig. 2(b), confirms that the $3 / 5$ island was removed, though a small residual $6 / 10$ island remains that was not targeted. The plasma stability and transport characteristics of the perturbed equilibrium have been analyzed and found to be unchanged from the original design. 


\section{Confinement}

The degree of quasi-axisymmetry can be characterized by the effective ripple strength $\varepsilon_{h, \text { eff }}$ [19], calculated numerically to match the 1/v transport regime. As shown in Fig. 3, the effective ripple rises exponentially to $\sim 3.4 \%$ at the plasma edge. The toroidal spectrum of the ripple is dominated by low-order perturbations, $n=3$ and 6 as shown in Fig. 4, which reduces its effect on confinement relative to typical tokamak ripple with $\mathrm{n} \sim 20$. This low $\varepsilon_{h, e f f}$, together with the relatively-high rotational transform results in acceptably low fast ion losses, as calculated by Monte-Carlo simulations [29] using the full 3D magnetic field. The calculated energy losses of $40 \mathrm{keV} \mathrm{H-neutral} \mathrm{beam} \mathrm{ions} \mathrm{with} \mathrm{B}=1.7 \mathrm{~T}$ is $\sim 15 \%$ for cotangential injection and $23 \%$ for counter-injection. These counter-injection losses are similar to those for a tokamak of similar size and are low enough that balanced neutral beam injection can be envisioned to control the beam-driven current and to allow control of rotation. The calculated alpha-particle losses in a projected reactor are $<20 \%$, including collisional effects, depending on the final size.

The thermal transport is assessed in two ways. The neoclassical confinement is calculated for specified profiles by Monte Carlo simulation using the GTC code [30]. The code simulates the full ion distribution function $(f)$ and the deviation of the electron distribution from a Maxwellian $(\delta f)$. It calculates the ambipolar electric field via a low-noise technique [31] for calculating the particle fluxes from the toroidal variation of $\mathrm{p}_{\|}+\mathrm{p}_{\perp}$. The second method combines models of the transport processes (helical neoclassical, toroidal neoclassical, anomalous) in a 1-D transport solver (STP) to predict temperature profiles and confinement for an assumed density profile. It includes an axisymmetric beam-deposition model and the 
Monte-Carlo code calculated fast-ion losses. The model for helical ripple-neoclassical transport uses the calculated $\varepsilon_{h, \text { eff }}$ and the Shaing-Houlberg full transport matrix [32]. Toroidal neoclassical transport is calculated folowing Chang-Hinton [33] normalized to an THRIFT/NCLASS [34] calculation averaging over the full 3D equilibrium. Anomalous transport is simulated using either a spatially uniform diffusivity or the Lackner-Gottardi model [35]. STP calculates the ambipolar electric field, choosing the ion root if it exists. The code can vary the magnitude of the anomalous transport model to match the total confinement time to a global confinement scaling, such as ISS-95 [36] or ITER-97P [37], times an enhancement factor. For a specified heating power, it will vary the density and confinement enhancement factor to achieve a specified $\beta$-value and collisionality, subject to an empirical density limit [38]. The two methods have been benchmarked and predict the same ambipolar electric field to within 5\% and the same ion energy flux within the MonteCarlo simulation uncertainty.

The predicted plasma transport is dominated by the anomalous transport and the toroidal neoclassical losses. The predicted ripple thermal transport is negligible in all cases studied. As an example, Fig. 5 shows the predicted radial power flow and temperature profiles for a $\beta=4 \%$ plasma with a collisionality $v^{*}=0.25$ at the half radius, using $\mathrm{B}=1.2 \mathrm{~T}, 6 \mathrm{MW}$ of neutral beam injected power, an average density of $6 \times 10^{19} \mathrm{~m}^{-3}$, and spatially uniform anomalous transport coefficients. Note that the ripple-neoclassical flux is insignificant and that the anomalous transport dominates over most of the profile. Simulations using the Lackner-Gottardi model give similar results. The calculations indicate that achieving $\beta=$ $4 \%$ with these parameters requires a global confinement time 2.9 times the ISS-95 scaling, somewhat higher than the best achieved on LHD and W7-AS. Since this configuration is designed to have tokamak-like drift orbits and the simulations predict tokamak-like trans- 
port, it is reasonable to compare this confinement to tokamak global scalings. If we use $I_{P}^{\text {Equiv }}$ to evaluate the tokamak scaling, the required confinement to achieve $\beta=4 \%$ and $v^{*}$ $=0.25$ is $\sim 0.9$ times the ITER-97P prediction. For comparison, similar sized PBX-M plasmas achieved $\beta=6.8 \%$ with $5.5 \mathrm{MW}$ of heating and $\mathrm{B}=1.1 \mathrm{~T}$ achieving a confinement of 1.7 times the ITER-97P prediction or $\sim 3.9$ times the ISS-95 prediction. Since ISS-95 and ITER-89P have different parametric dependencies, the confinement multipliers must be expected to vary separately. For the same conditions, except $B=1.7 \mathrm{~T}$, sTP predicts central temperatures of $2.3 \mathrm{keV}, \mathrm{v}^{*}=0.1$.

There are a number of reasons to expect that the confinement may be enhanced in this configuration, relative to standard stellarators and tokamaks. The high degree of quasisymmetry should reduce flow damping, allowing development of persistent zonal flows. This may stabilize turbulence at lower levels than in non-symmetric stellarators. In addition, the 'reversed' shear should stabilized trapped particle modes and eliminate the trappedelectron drive for ITG turbulence $[39,40]$, similar to reversed-shear advanced tokamak regimes.

\section{Coil Design \& Flexibility}

A wide range of coil topologies and designs has been explored for generating the equilibrium in Fig. 1, including modular, helical, and saddle coils, using a number of optimization strategies [41]. Of these, the modular coils (shown in Fig. 6) best reproduce the physics properties and good flux surfaces of the target equilibrium. These coils would be used with a set of poloidal field coils, to control plasma position and average shape, and a weak toroidal solenoid to allow variation of rotational transform. The modular coil design shown has 7 
coils per period, with 4 different coil shapes. The coils at the elongated symmetry plane have been extended radially to allow tangential neutral beam injection and to provide tangential diagnostic views. The coils do not reproduce the original fixed-boundary plasma shape precisely, but approximate it subject to engineering constraints on coil characteristics, such as bend radii and coil-separation distances. To test the adequacy of a coil design, the coil currents are re-optimized to achieve the original plasma criteria (MHD stability and quasi-axisymmetry) based on free-boundary equilibrium calculations. Free-boundary optimized equilibria have been found that reproduce the key properties of original fixedboundary design.

The coils must not introduce large islands or stochastic field regions. The coil shapes are perturbed to remove resonant fields that produce islands, as calculated in a free-boundary PIES equilibrium. The algorithm is similar to that used for removing the fixed-boundary equilibrium islands, above. The resulting coils are calculated to be able to produce equilibria with good flux surfaces over a wide range of conditions, as shown in Fig.7.

For an experiment, the coil system must robustly handle a variety of pressure and current profiles and be flexible to handle the discharge evolution and generate a variety of configurations for physics studies. This has been studied using the free-boundary optimizer to test whether a coil design can produce equilibria with specified properties. For these studies, it is assumed that the current in each coil type can be independently controlled (preserving stellarator symmetry). Figure 8 shows a study of the accessible range of edge rotational transform with the modular coils, for fixed pressure and plasma current, while optimizing quasi-axisymmetry and constraining the plasma to be within a design vacuum vessel. For the cases shown, the ripple magnitude is no more than 1.8 times the original optimized con- 
figuration. A wide range of operation is available, including configurations where the transform profile is entirely above or below $1 / 2$. This flexibility will allow control of the edge rotational transform, if needed, preventing it from passing through $1 / 2$ during the discharge evolution, thus avoiding the tearing modes and disruptions observed in hybrid operation of W7-AS [3]. A similar study varied the magnetic shear down to approximately shearless at full plasma current by varying the modular coil currents. In these cases, the quasi-symmetry was degraded by up to a factor of 5 (for the shearless case). A set of trim coils is planned to control low-order resonant field components and the islands they generate, to allow operation over a large range of magnetic transform profiles.

Accessible free-boundary equilibria have been found with substantially improved or degraded quasi-axisymmetry, for fixed plasma profiles and $\mathfrak{t}$ profile. Similarly, the kink-beta limit can be varied by at least a factor of 3 just by varying the plasma shape via external coil currents, either at fixed edge $\mathfrak{t}$ or with a fixed shear profile. This ability to manipulate the plasma characteristics will enable controlled experiments for comparison with theoretical predictions and testing models. Surprisingly, the $\beta$-limit for free-boundary equilibria has not been found yet. Stable equilibria with $\beta>6.5 \%$ have been found with some degradation of the quasi-symmetry, but without yet making use of profile optimization.

The evolution of the plasma current from vacuum through an Ohmic current-ramp to equilibration with the bootstrap current has been simulated using an assumed temperature evolution. By assuming early auxiliary heating to increase the temperature, as used in reversedshear tokamak experiments, broad current profiles were predicted which equilibrate with the bootstrap current in $\sim 0.4 \mathrm{sec}$. The current evolution was approximated using an axisymmetric calculation, representing the rotational transform from the coils as a constant imposed 
external current drive profile. The calculated current profiles and pressure profiles were used with the free-boundary optimizer to calculate the evolution of coil-currents constraining the plasma shape to stay approximately fixed. Simulation of the evolution from vacuum to $\beta=4.25 \%$ showed reasonable coil-current variations and that kink-modes were calculated to be stable throughout the evolution. Stable evolution scenarios have been found where $\mathfrak{t}(\mathrm{a})$ either crosses $1 / 2$ or is always above $1 / 2$. In all cases studied, $\Delta^{\prime}$ analysis indicates that the current profiles are stable or marginally stable to tearing instabilities.

In simulations of unidirectional tangential neutral beam injection, the beam driven current strongly changed the core rotational transform. For co-tangential only injection, the central rotational transform rapidly goes above one, producing a tokamak-like shear profile, which is unstable to neoclassical tearing modes. From these simulations, balanced co- and counterinjection will be required to obtain the optimized current profile. Variations away from balanced injection could be used to control the central magnetic shear.

\section{Conclusions}

A novel compact quasi-axisymmetric stellarator has been designed for NCSX, combining features from optimized stellarators and advanced tokamaks, and offering a possible path to steady-state reactors without current drive or disruptions. Extra design flexibility from 3D shaping has been used to passively stabilize the kink, vertical, ballooning, Mercier, and neoclassical tearing modes at $\beta>4 \%$ without need for external conducting walls or feedback, while maintaining good orbit confinement. The calculated confinement characteristics are similar to an equivalent tokamak. This NCSX design demonstrates the power of the recent 
advances in experimental and theoretical understanding and numerical modeling, and illustrates the possibilities available for magnetic confinement with 3D shaping.

\section{Acknowledgements}

We gratefully acknowledge useful discussions with P. Garabedian , C. Hegna, H. Maassberg, and J. Nuehrenberg. This research was supported by the US Department of Energy under contracts DE-AC-76-CH0-3073 and DE-AC05-00OR22725, by Euratom, and by the Fonds National Suisse de la Recherche Scientifique. 


\section{Figures Captions:}

FIG. 1. Plasma boundary shape in four poloidal cross-sections separated $20^{\circ}$ toroidally.

FIG. 2. Poincare plot of flux-surface structure: (a) original target plasma of Fig. 1, (b) with boundary perturbed to remove islands(fewer flux surfaces plotted)

FIG. 3. Radial profile of effective helical ripple.

FIG. 4. Radial profile of non-axisymmetric components of the magnetic field strength $|\mathrm{B}|$ in Boozer coordinates.

FIG. 5. (a) Predicted $\mathrm{T}_{\mathrm{e}}$ and $\mathrm{T}_{\mathrm{i}}$ profiles, and (b) Radial power flows for $\mathrm{B}=1.2 \mathrm{~T}, \mathrm{P}=6 \mathrm{MW}$.

FIG. 6. Optimized modular coils for the equilibrium of Fig.1 .

FIG. 7. Poincare plots of flux-surface structure for free boundary equilibria: (a) vacuum, (b) $\beta=2 \%, I_{P}=83 \mathrm{kA}$, (c) $\beta=4.1 \%, I_{P}=125 \mathrm{kA}$. Current values correspond to $B=1.2 \mathrm{~T}$.

FIG. 8. Free-boundary optimized variations of the rotational transform profile using modular coils, maintaining approximate quasi-symmetry, with fixed plasma pressure and current profiles. 


\section{References}

[1] W VII-A Team, Nucl. Fusion 20 (1980) 1093.

[2] D.C. Robinson and T.N. Todd, Phys. Rev. Letters 48 (1982) 1359.

[3] A. Weller et al, IAEA 2000; E. Sallander, A. Weller, and W7-AS Team, Nucl. Fusion 40 (2000) 1499.

[4] J. Nührenberg and R. Zille, Phys. Lett. A 114 (1986) 129.

[5] C. Beidler et al., Fusion Technol. 17 (1990) 148.

[6] L.M. Kovrizhnykh, Nucl. Fusion 25 (1985) 1391.

[7] H. Wobig, Plasma Phys. Control. Fusion 41 (1999) A159.

[8] D. Spong et al, Nucl. Fusion 41 (2001) 711.

[9] A. Boozer, Phys. Fluids 26 (1983) 496.

[10] J. Nührenberg and R. Zille, Phys. Lett. A 129 (1988) 113.

[11] J. Nührenberg, W. Lotz, and S. Gori, in Theory of Fusion Plasmas (E. Sidoni et al., Eds.) (SIF, Bologna, 1994)

[12] P. Garabedian, Phys. Plasmas 3 (1996) 2483.

[13] D. Anderson et al, paper P2.076 this conference.

[14] M.N. Rosenbluth and F.L. Hinton, Phys. Rev. Letters 80 (1998) 724.

[15] S. Okamura et al., IAEA 2000 (Sorrento).

[16] S.P. Hirshman and J.C. Whitson, Phys. Fluids 26 (1983) 3533.

[17] D.V. Anderson et al., Int. J. Supercomput. Appl. 4 (1990) 34.

[18] R. Sanchez et al., J. Comp. Phys. 161 (2000),589.

[19] V.V. Nemov et al., Phys. Plasmas 6 (1999) 4622.

[20] S.P Hirshman et al, Phys. Fluids 29 (1986) 2951; W.I. van Rij and S.P. Hirshman, Phys. Fluids B1 (1989) 563.

[21] P. Merkel, Nucl. Fusion 27 (1987) 867.

[22] G.Y. Fu et al., Phys. Plasmas 7 (2000) 1809.

[23] G.Y. Fu et al., IAEA 2000 Sorrento.

[24] A. Reiman et al., Plasma Phys. Control. Fusion 41(1999) B273.

[25] G.H. Neilson et al., Phys. Plasmas 7 (2000) 1911.

[26] A.H. Reiman and H.S. Greenside, J. Comput. Phys. 75 (1988) 423.

[27] S.R. Hudson, D. A. Monticello, and A.H. Reiman, Phys. Plasmas 8 (2001), accepted for publication in the July issue.

[28] S.R. Hudson and R.L. Dewar, Phys. Plasmas 6 (1999) 1532.

[29] D.A. Spong et al., Fusion Energy 1998 (Proc. 17 ${ }^{\text {th }}$ Int. Conf. Yokohama, 1998), (IAEA, Vienna, 1999), Vol. 3, 1159.

[30] Z. Lin et al., Phys. Plasmas 2 (1995) 2975.

[31] J. Lewandowski et al, Phys. Plasmas 8 (2001) 2849.

[32] D.E. Hastings et al., Nucl. Fusion 25 (1985) 445.

[33] C.S. Chang and F.L. Hinton, Pys. Fluids 29 (1986) 3314.

[34] P.I. Strand and W.A. Houlberg, Phys. Plasmas 8 (2001) 2782.

[35] K. Lackner and N.A.O. Gottardi, Nucl. Fusion 30 (1990) 767.

[36] U. Stroth et al., Nucl. Fusion 36 (1996) 1063. 
[37] S. Kaye et al, Nucl. Fusion 37 (1997) 1303.

[38] S. Sudo et al., Nucl. Fusion 30 (1990) 11.

[39] C. Kessel et al, Phys. Rev. Lett. 72 (1994) 1212.

[40] M. Yokoyama et al, Phys. Rev. E 64 (2001) 15401.

[41] N. Pomphrey et al, Nucl. Fusion 41 (2001) 339. 
Fig. 1

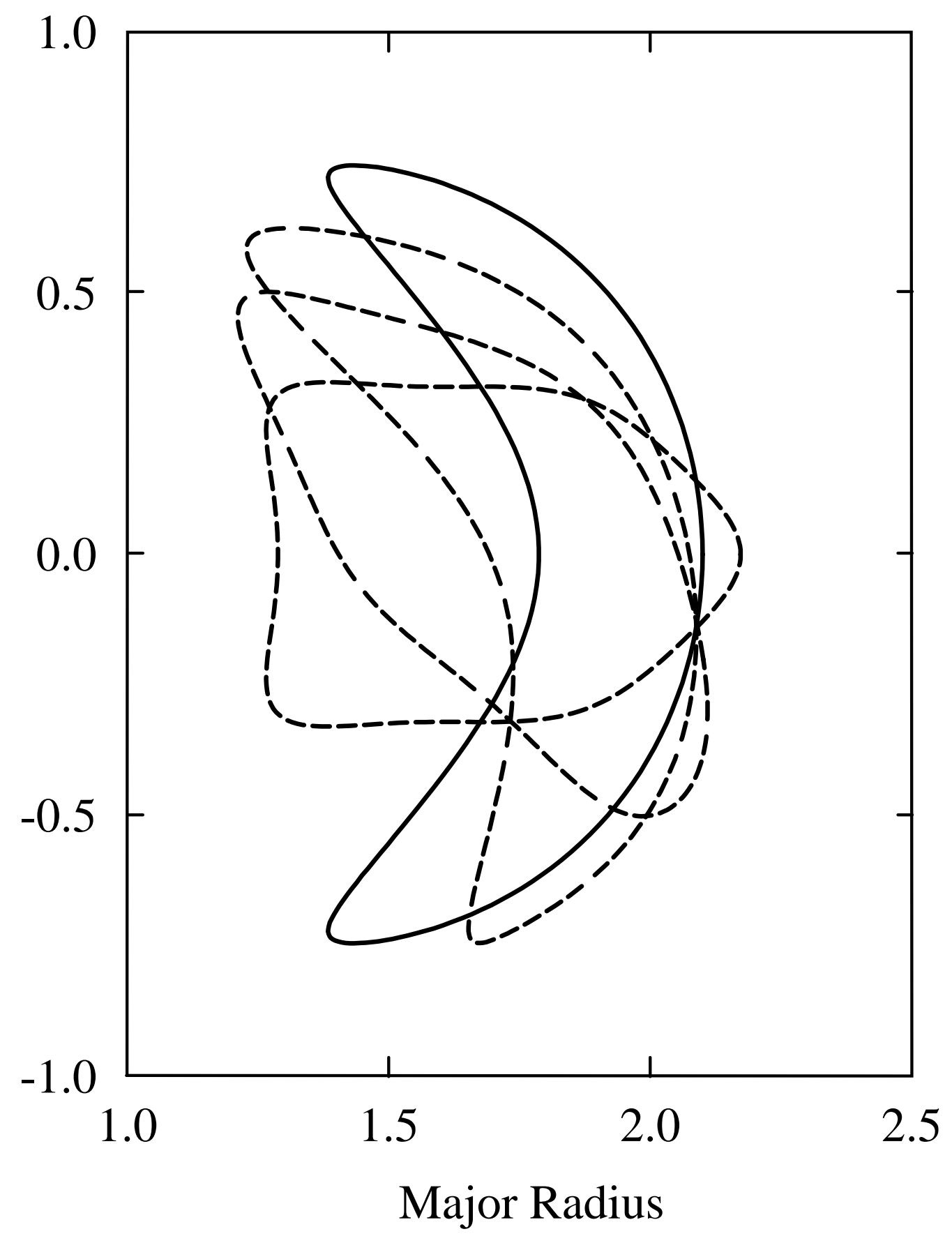



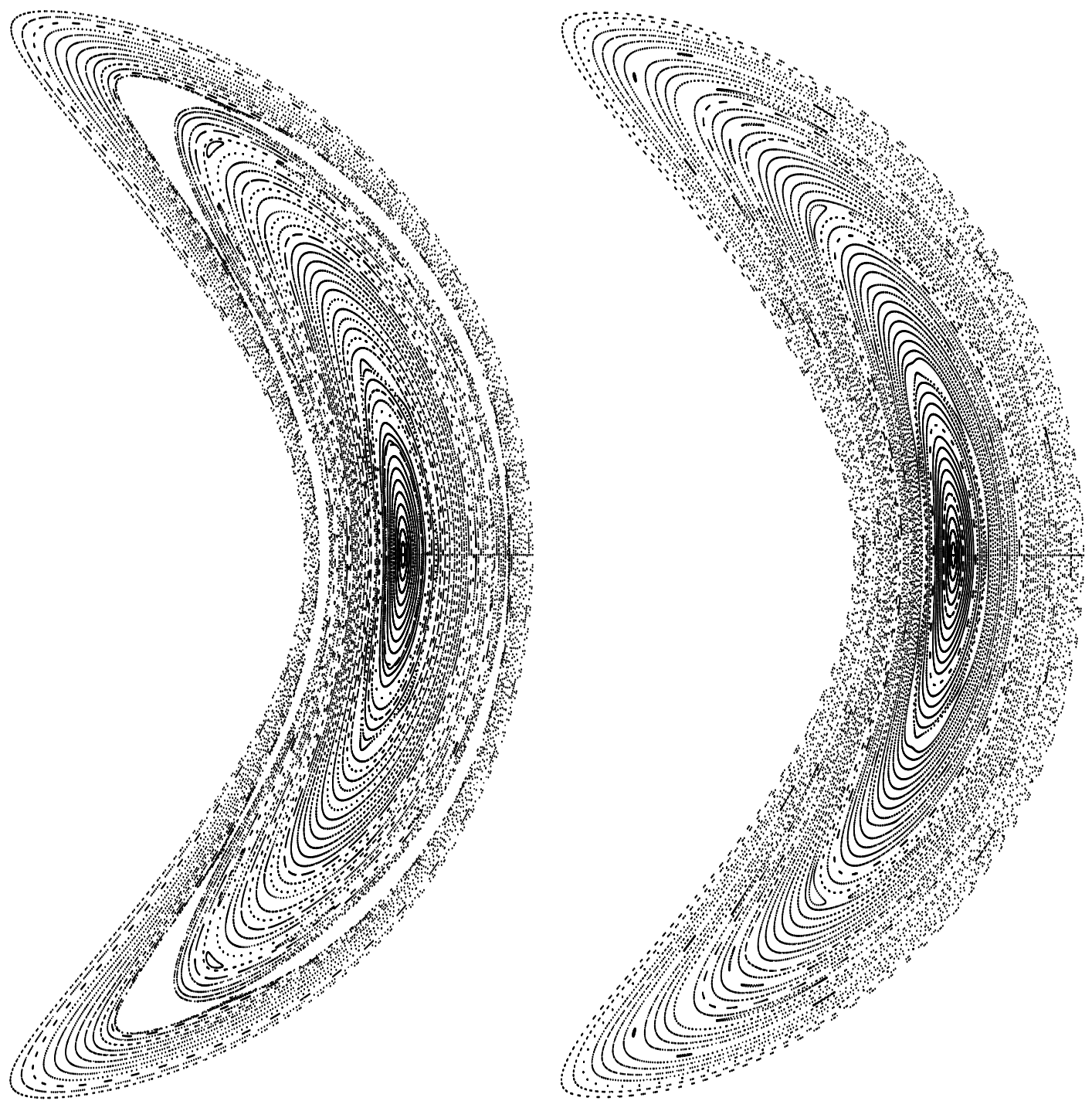

Fig. 2 


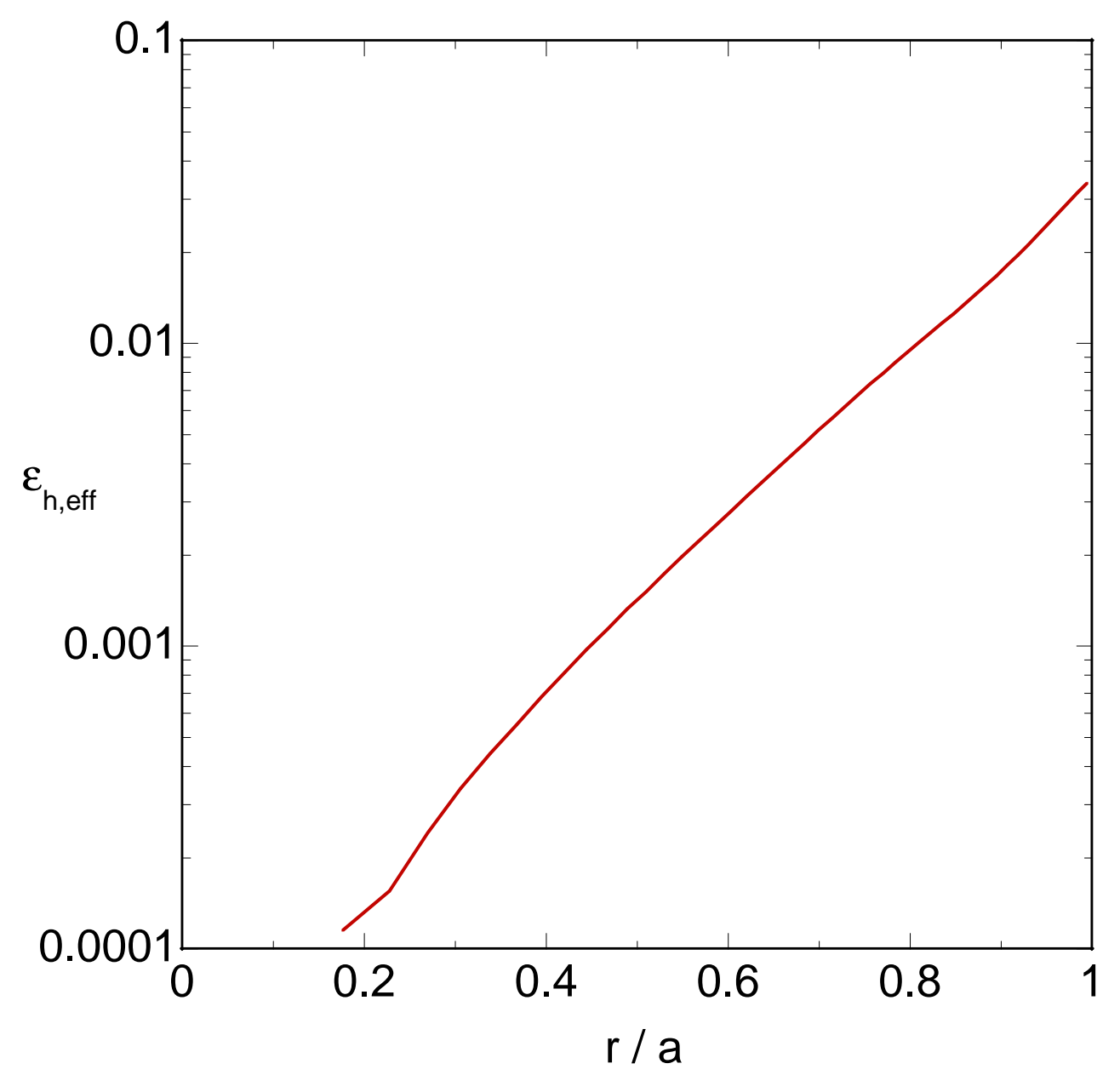

Fig. 3 
क़०

$A$

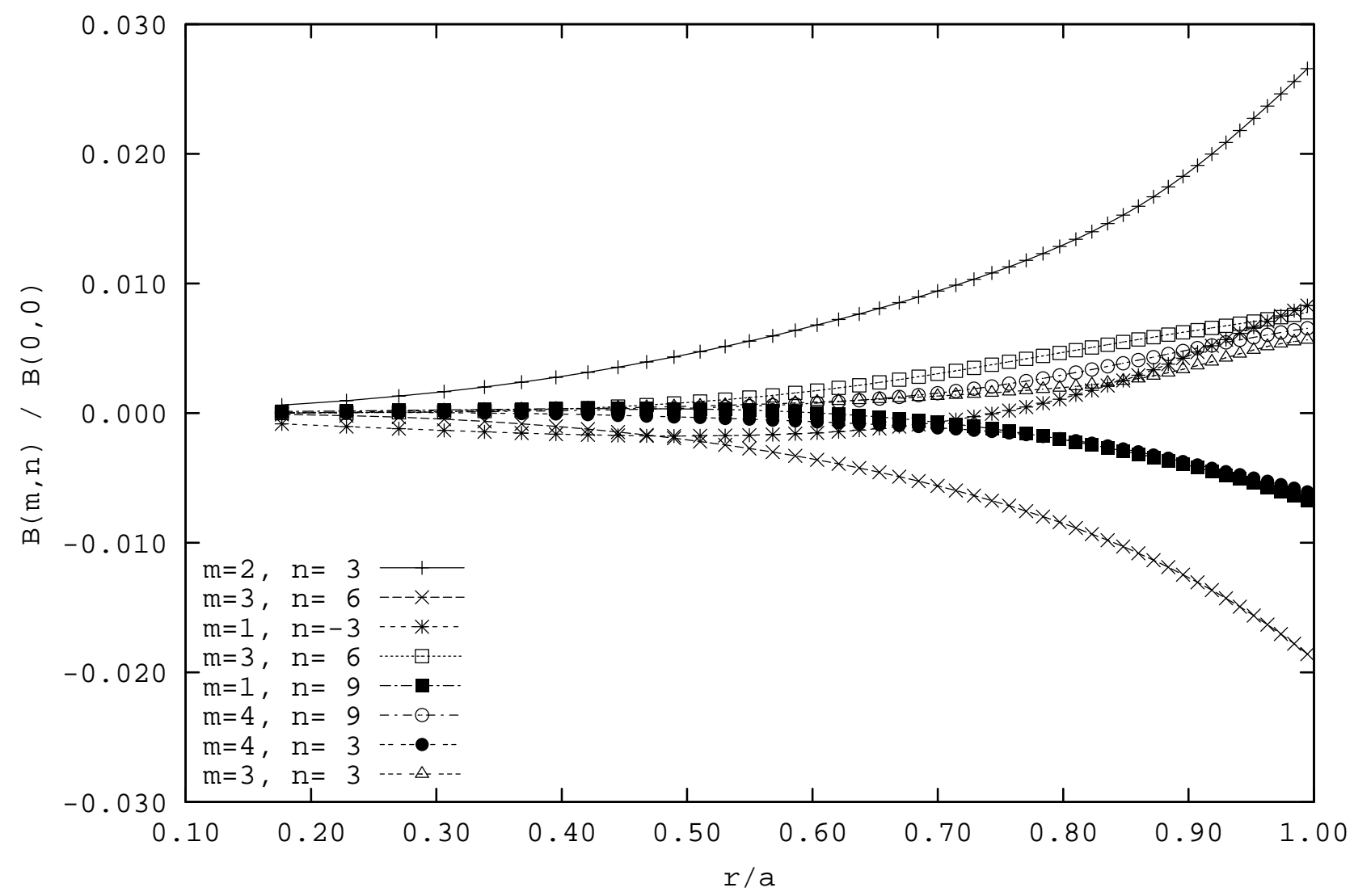



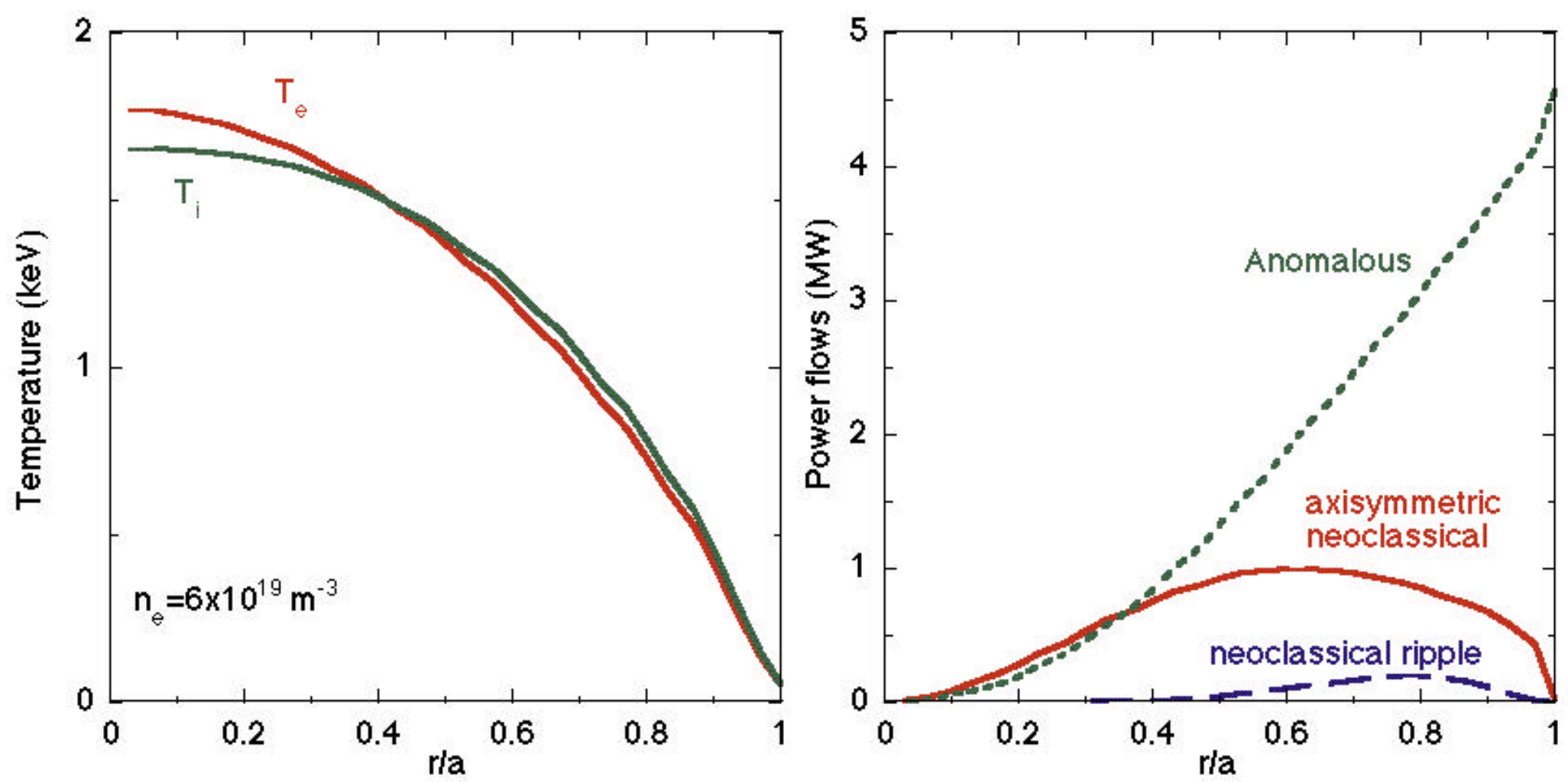

Fig. 5 


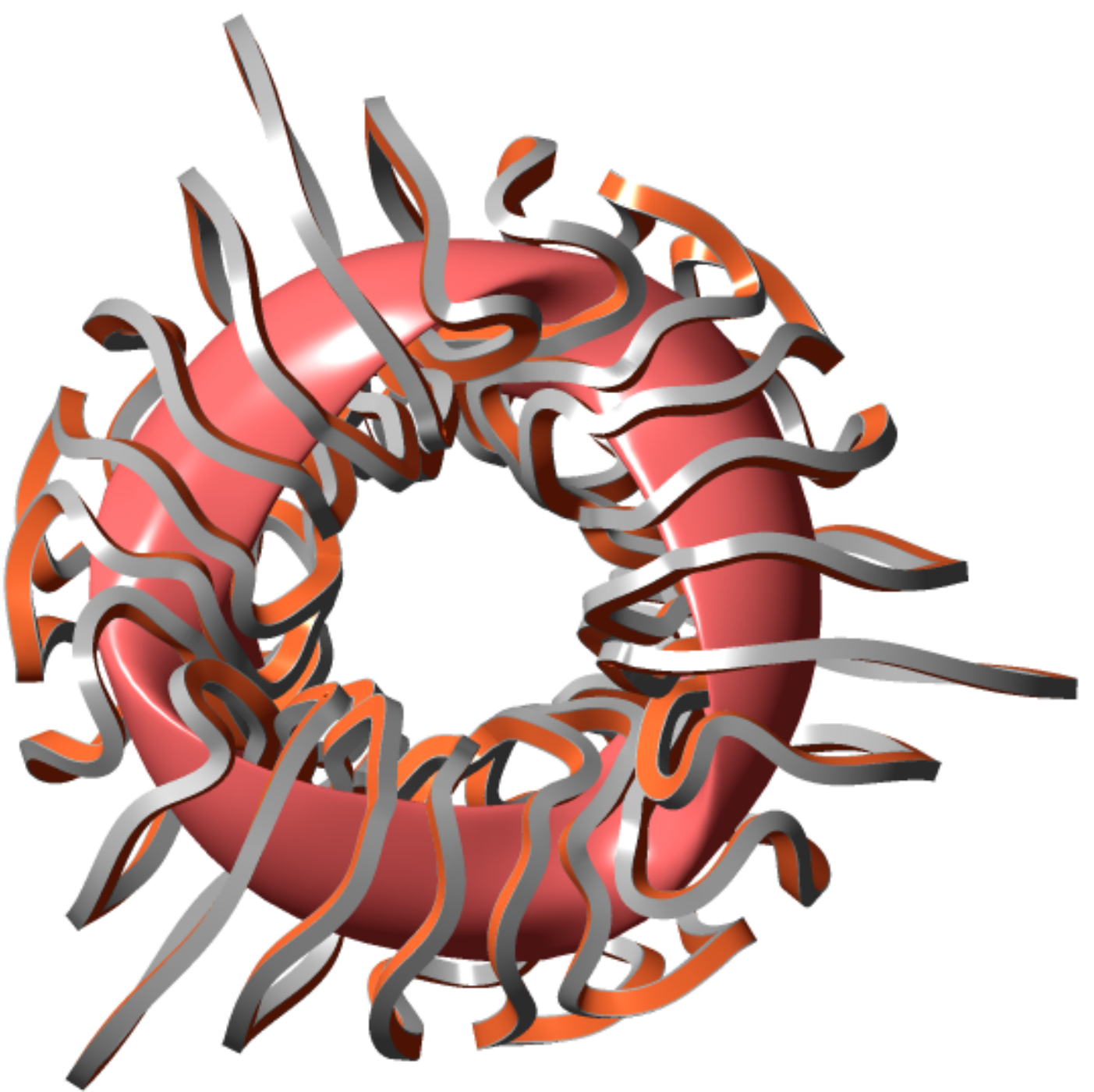

Fig. 6 

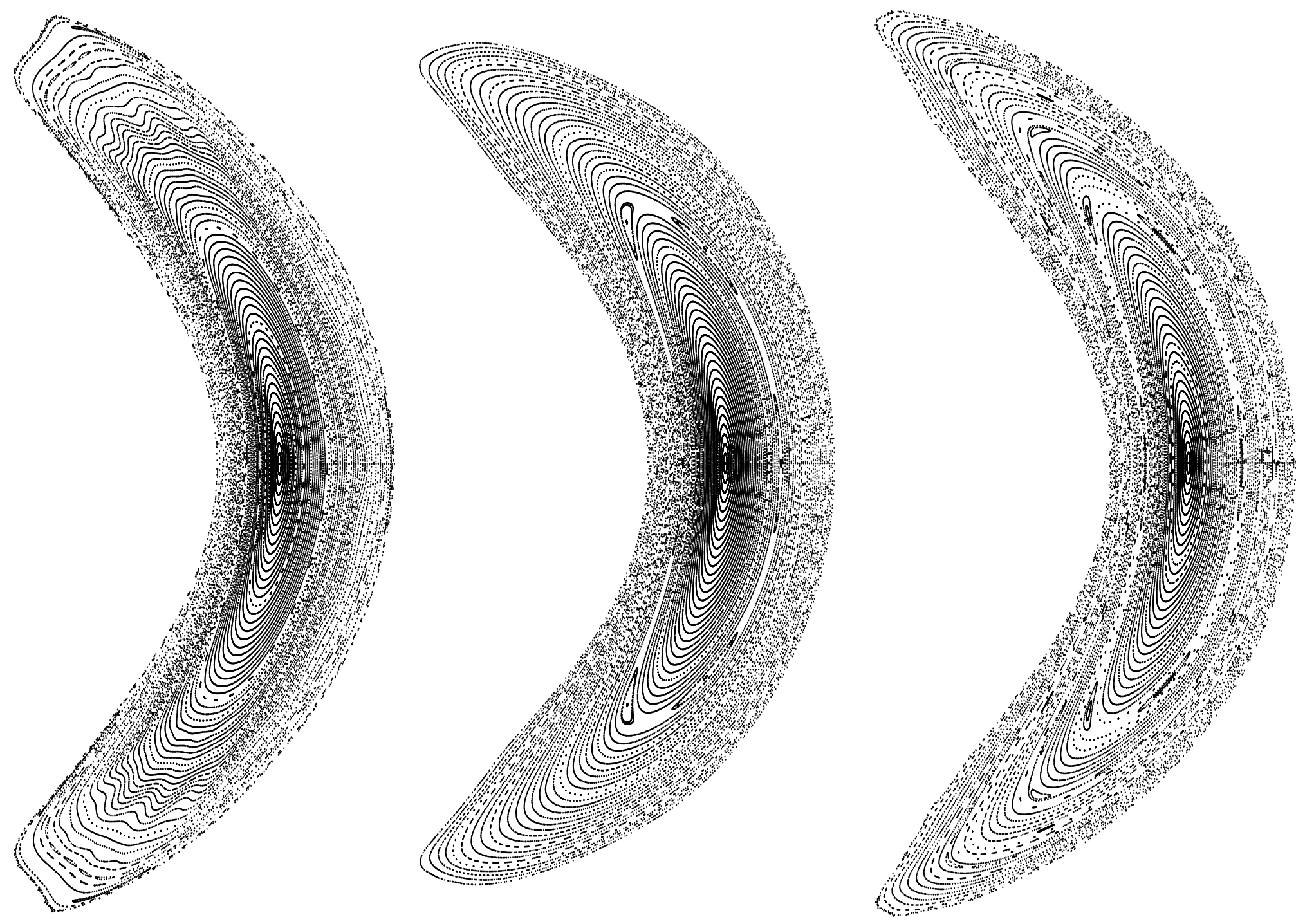

Fig. 7 


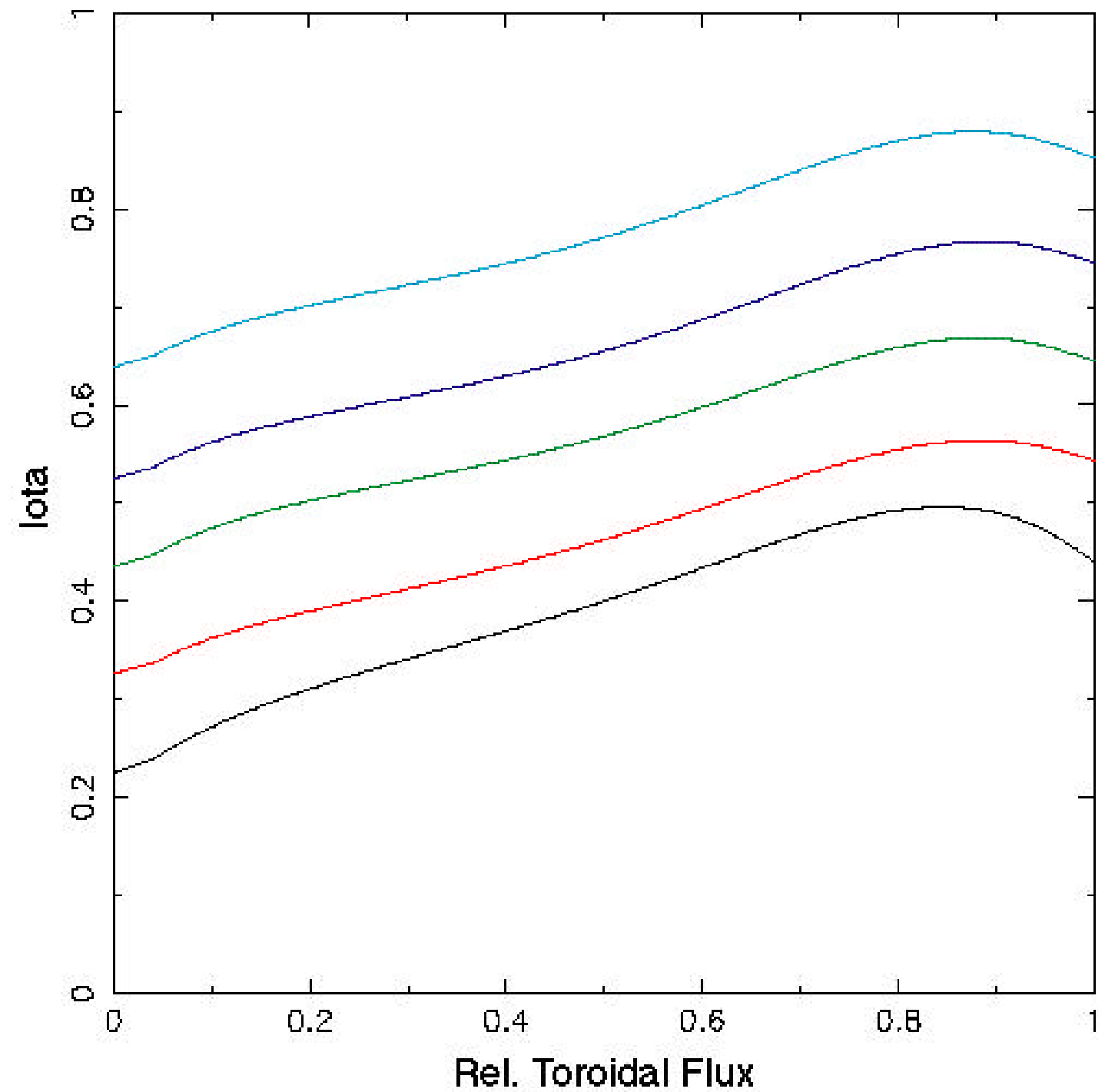

Fig. 8 


\section{External Distribution}

Plasma Research Laboratory, Australian National University, Australia

Professor I.R. J ones, Flinders University, Australia

Professor J oão Canalle, Instituto de Fisica DEQ/IF - UERJ , Brazil

Mr. Gerson O. Ludwig, Instituto Nacional de Pesquisas, Brazil

Dr. P.H. Sakanaka, Instituto Fisica, Brazil

The Librarian, Culham Laboratory, England

Library, R61, Rutherford Appleton Laboratory, England

Mrs. S.A. Hutchinson, JET Library, England

Professor M.N. Bussac, Ecole Polytechnique, France

Librarian, Max-Planck-Institut für Plasmaphysik, Germany

J olan Moldvai, Reports Library, MTA KFKI-ATKI, Hungary

Dr. P. Kaw, Institute for Plasma Research, India

Ms. P.J . Pathak, Librarian, Insitute for Plasma Research, India

Ms. Clelia De Palo, Associazione EURATOM-ENEA, I taly

Dr. G. Grosso, Instituto di Fisica del Plasma, Italy

Librarian, Naka Fusion Research Establishment, J AERI, J apan

Library, Plasma Physics Laboratory, Kyoto University, J apan

Research Information Center, National Institute for Fusion Science, J apan

Dr. O. Mitarai, Kyushu Tokai University, J apan

Library, Academia Sinica, Institute of Plasma Physics, People's Republic of China

Shih-Tung Tsai, Institute of Physics, Chinese Academy of Sciences, People's Republic of China

Dr. S. Mirnov, TRINITI, Troitsk, Russian Federation, Russia

Dr. V.S. Strelkov, Kurchatov Institute, Russian Federation, Russia

Professor Peter Lukac, Katedra Fyziky Plazmy MFF UK, Mlynska dolina F-2, Komenskeho Univerzita, SK-842 15 Bratislava, Slovakia

Dr. G.S. Lee, Korea Basic Science Institute, South Korea

Mr. Dennis Bruggink, Fusion Library, University of Wisconsin, USA

Institute for Plasma Research, University of Maryland, USA

Librarian, Fusion Energy Division, Oak Ridge National Laboratory, USA

Librarian, Institute of Fusion Studies, University of Texas, USA

Librarian, Magnetic Fusion Program, Lawrence Livermore National Laboratory, USA

Library, General Atomics, USA

Plasma Physics Group, Fusion Energy Research Program, University of California at San Diego, USA

Plasma Physics Library, Columbia University, USA

Alkesh Punjabi, Center for Fusion Research and Training, Hampton University, USA

Dr. W.M. Stacey, Fusion Research Center, Georgia Institute of Technology, USA

Dr. J ohn Willis, U.S. Department of Energy, Office of Fusion Energy Sciences, USA

Mr. Paul H. Wright, Indianapolis, Indiana, USA 
The Princeton Plasma Physics Laboratory is operated by Princeton University under contract with the U.S. Department of Energy.

\author{
Information Services \\ Princeton Plasma Physics Laboratory \\ P.O. Box 451 \\ Princeton, NJ 08543
}

Phone: 609-243-2750

Fax: 609-243-2751

e-mail: pppl_info@pppl.gov

Internet Address: http://www.pppl.gov 